\title{
Visual Scale Factor for Speed Perception
}

\author{
Florent Colombet ${ }^{1}$, Damien Paillot ${ }^{1}$, Frédéric Mérienne ${ }^{1}$, Andras Kemeny ${ }^{1,2}$ \\ 1 : Arts et Metiers ParisTech, CNRS, Le2i, Institut Image \\ 2 rue Thomas Dumorey, 71100 Chalon-sur-Saône, France \\ 2 : Technical Center for Simulation, Renault \\ 1 avenue du Golf, 78288 Guyancourt
}

colombet@cluny.ensam.fr

merienne@cluny.ensam.fr

paillot@cluny.ensam.fr

andras.kemeny@renault.com

\section{Abstract}

Speed perception is an important task depending mainly on optic flow that the driver must perform continuously to control his/her vehicle. Unfortunately it appears that in some driving simulators speed perception is under estimated, leading into speed production higher than in real conditions. Perceptual validity is then not good enough to study driver's behavior. To solve this problem, a technique has recently seen the light, which consists of modifying the geometric field of view (GFOV) while keeping the real field of view (FOV) constant. We define our visual scale factor as the ratio between the GFOV and the FOV.

The present study has been carried out on the SAAM dynamic driving simulator and aims at determining the precise effect of this visual scale factor on the speed perception. 20 subjects have reproduced 2 speeds $(50 \mathrm{~km} / \mathrm{h}$ and $90 \mathrm{~km} / \mathrm{h})$ without knowing the numerical values of these consigns, with $\mathbf{5}$ different visual scale factors: $0.70,0.85,1.00,1.15$ and 1.30 . We show that speed perception significantly increases when the visual factor increases. A 0.15 modification of this factor is enough to obtain a significant effect. Furthermore, the relative variation of the speed perception is proportional to the visual scale factor.

Besides, the modification of the geometric field of view remained unnoticed by all the subjects, which implies that this technique can be easily used to make drivers reduce their speed in driving simulation conditions. However, this technique may also modify perception of distances.

\section{Introduction}

Driving simulation allows road safety institutions or car manufacturers to study driver's behavior. Some want to obtain a better understanding of accident situations; others want to test the ergonomics, the safety or new driving aid systems of their future cars with digital prototypes and drivers in the loop. However in both cases drivers have to be provided with motion cues as close as possible with those in real conditions in order to ensure their behavior fidelity. That's why a large number of human factors studies (Kemeny \& Panerai, 2003) (Kennedy et al, 1993) have been carried out with driving simulators to study driver behavior or self-motion perception in driving conditions.

In this paper we will investigate more precisely speed perception, which is one of the most crucial skills and a constant demand that the driver must perform. 
(Kemeny \& Panerai, 2003) review the many factors influencing evaluation of vehicle speed: visual cues such as optic flow, time-to-contact, field of view, angular declination, image contrast or weather conditions but also vestibular, audio, kinesthetic and proprioceptive cues (Berthoz, 1995). Some authors ((Gibson \& Crooks, 1938), (Gibson, 1979), (Lappe et al, 1999)) have studied in-depth the influence of visual cues on speed perception. They focused particularly on optic flow, i.e. the variation of the retina image, and show that it is one of the main cues used for the evaluation of speed. (Berthoz et al, 1975) have also shown the importance of peripheral vision for the perception of linear horizontal self-motion. According to (Jamson, 2000), the image resolution and the field of view have a significant impact on speed perception. (Panerai et al, 2001) also showed the significant influence of the height of the driver viewpoint on the perception of speed in comparison to realworld driving.

It has been reported that speed is often under-estimated in virtual environments and in driving simulators ((Blaauw, 1982) (Banton, et al, 2005)). We may explain this by the fact that all the different sensory cues involved in the evaluation of speed are not always rendered in these systems. In driving simulators for example, the lack of car vibrations rendering or a small field of view could lead to a wrong perception of speed: speed overproductions are then observed (Mourant et al, 2007). Unfortunately in this case, driver's behavior can be affected in tasks like cornering or emergency braking. Perceptual validity is then not good enough to study driver's behavior.

To solve this problem, (Diels \& Parkes, 2009) have developed a technique based on the results of (Mourant et al, 2007). They change the geometric field of view (GFOV) i.e. the field of view of the virtual camera, while keeping the real field of view (FOV) constant. By this way they manage to modify driver's speed perception without being noticed. In both studies, authors find that perceived speed increases when the geometric field of view increases. However two questions remain unresolved. First why the GFOV impacts the perceived speed? Neither Diels or Mourant provide much explanation about this. Secondly what is the exact relationship between the variation of GFOV and the variation of perceived speed? We propose here to investigate these two questions.

\section{Geometric field of view and perceived speed}

(Mourant et al, 2007) studied the influence of the geometric field of view on speed perception. In their experiment, they asked subjects to produce certain speeds (30 and $60 \mathrm{mph}$ ) on a static driving simulator with a 45 deg curved screen and with different geometric field of view conditions (25,55 and $85 \mathrm{deg}$ ). They found that produced speed is highly correlated to the GFOV and that produced speed decreases when the GFOV increases. (Diels \& Parkes, 2009) made a similar experiment on the TRL driving simulator (visual display covering 210 deg, vibrations rendering, no motion). Subjects were also asked to produce different speeds (20, 30, 50 and $70 \mathrm{mph}$ ) with four different GFOV conditions (175, 210, 245 and $280 \mathrm{deg}$ ). They obtained similar results. Moreover, in (Diels \& Parkes, 2009) experiment, subjects never reported having seen visual variations during the experiment. In order to compare the results and as the screen sizes of all simulators are not the same, 
(Diels \& Parkes, 2009) propose to take as an input the ratio GFOV/FOV instead of directly the GFOV. We have chosen to call this ratio the visual scale factor.

In both experiments, how the visual scale factor affects the perceived speed is not explained. In order to understand this we have first to know how the GFOV is modified. (Diels \& Parkes, 2009) explain that the easiest way to increase or decrease the GFOV is to move the position of the virtual camera (Figure 1.A). Because the virtual and the real screens stay joined and also because the driver stays at the same position relatively to the real screen, the geometric field of view is modified while the real field of view is kept constant. Thus the visual scale factor is increased (respectively decreased) when the virtual point of view is moved forward (respectively backward).

To explain the impact of the geometric field of view on the perceived speed, our hypothesis is that the optic flow is altered by the visual scale factor ( $\left.f_{v s}\right)$. Figures 1.B, 1.C and 1.D show the projection on the screen of the same velocity vector $\vec{V}$ in 3 cases: with a visual factor of 1.0, greater than 1.00 and smaller than 1.0. In the first case (Figure 1.B), GFOV and FOV are equals so virtual and real points of view are joined. The perceived speed $\overrightarrow{\widetilde{V}}$ is then equal to $\vec{V}$. When the visual scale factor is greater than 1.0 (Figure 1.C), the virtual point of view is moved forward and $\vec{V}$ is displayed on the screen in $\overrightarrow{V_{A}^{\prime}}$. The perceived speed $\left\|\overrightarrow{\vec{V}_{A}}\right\|$ is then greater than $\|\vec{V}\|$. Conversely when the visual scale factor is smaller than 1.00 (Figure 1.D) the virtual point of view is moved backward, $\vec{V}$ is displayed on the screen in $\overrightarrow{V_{B}^{\prime}}$ and the perceived speed $\left\|\vec{V}_{B}\right\|$ is then smaller than $\|\vec{V}\|$.

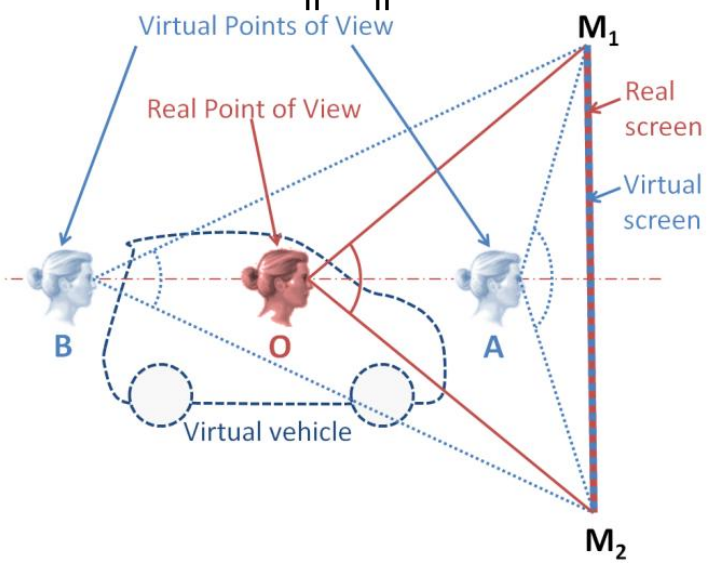

A

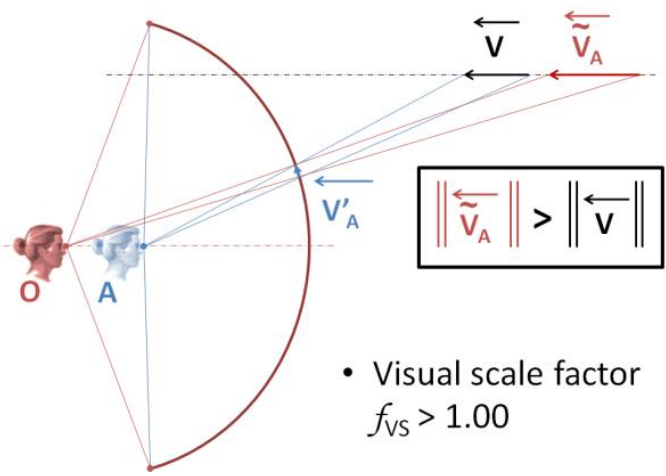

C

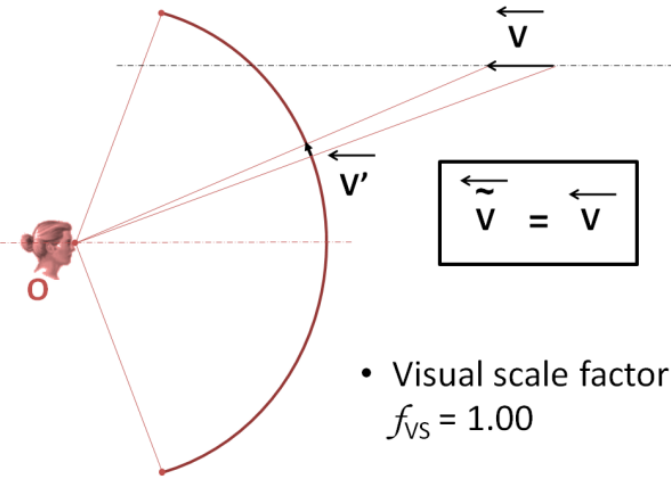

B

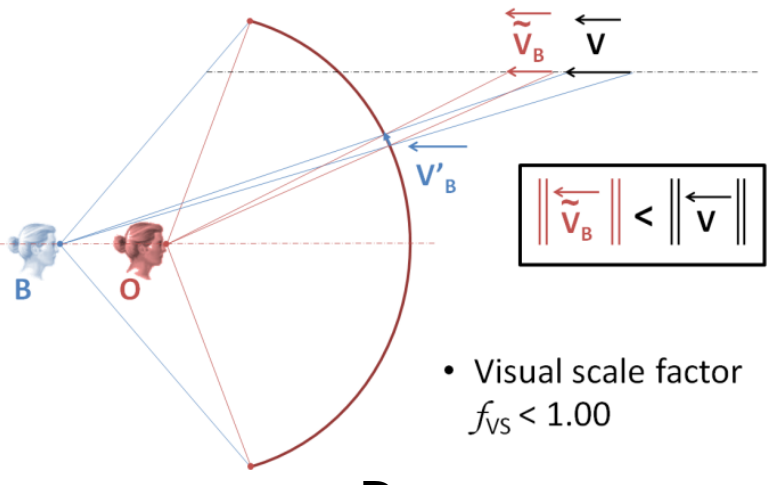

D

Figure 1 - Relations between the geometric field of view and the perceived speed. 
The optic flow seems to be modified by the visual scale factor and could thus explain why perceived speed is affected by this technique. The Figure 2 shows the optic flow experienced by an observer moving in the direction of a vertical plan. This optic flow has been computed with different visual scale factors $(0.50,0.75,1.00$, 1.25 and 1.50$)$. We clearly see that perception of speed is greater with $f_{V S}=1.50$ than with $f_{V S}=0.50$ : the length of the same speed vectors increases when the visual scale factor increases.
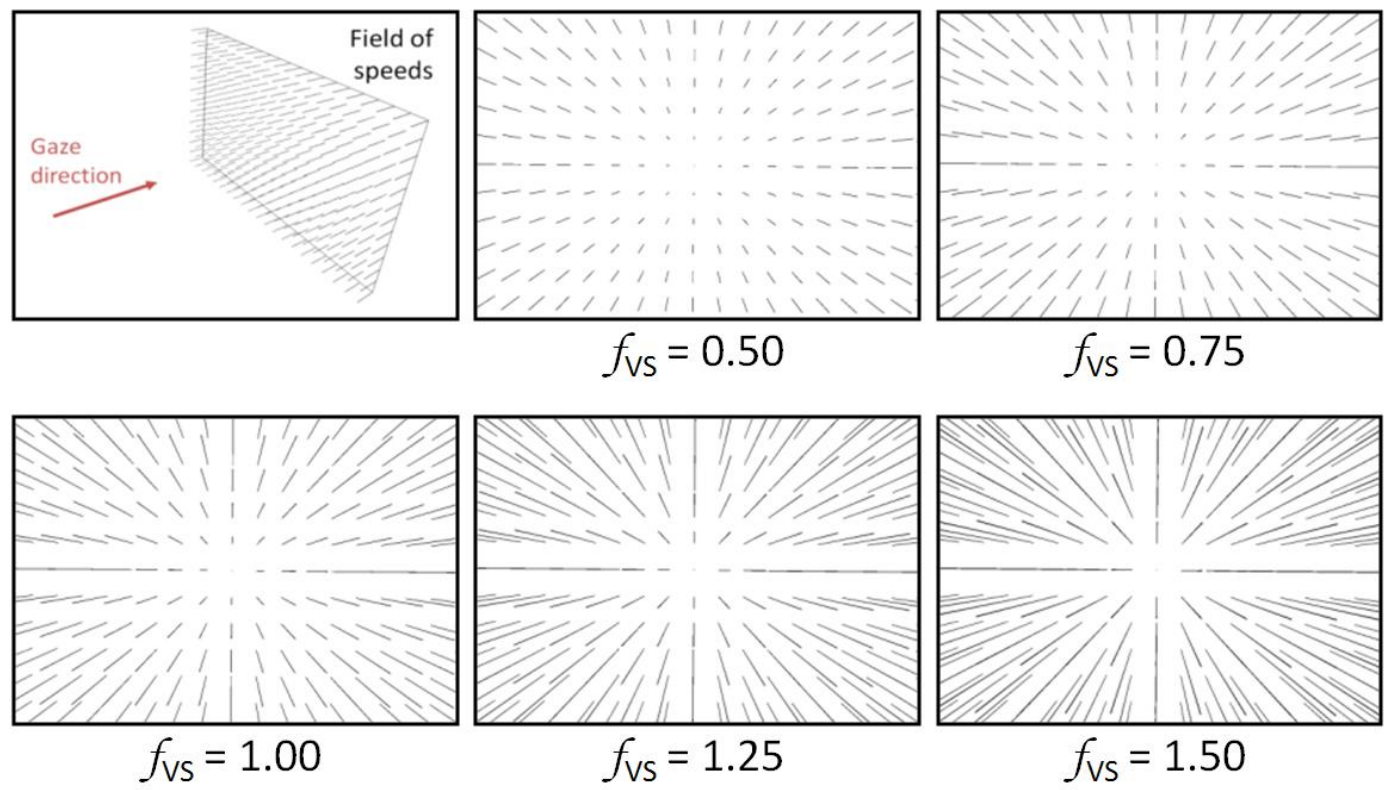

Figure 2 - Images of the same field of speeds computed with different visual scale factors.

\section{Experimental study}

(Mourant et al, 2007) and (Diels \& Parkes, 2009) both claim that perceived speed increases with the size of the GFOV but what remains unanswered is the exact relationship between the variation of the visual scale factor and the variation of perceived speed. The experimental study presented here will answer this question.

Besides, we will try to get rid of the bias inherent to the speed production task. Actually we think that when subjects are asked to produce a certain speed they are likely to reproduce the speed sensation they experience in the car they usually drive. And as cars do not all provide the same speed sensation because their height or the field of view they provide can differ, different subjects will not produce the same speed even if the speed consign is the same. That's why we propose to ask subjects to reproduce instead of produce different speeds, in order to never let them know the numerical value of their virtual vehicle speed.

\section{Participants}

Twenty volunteer subjects ( 3 female and 17 male) external to the lab participated in this study. They ranged in age from 20 to 69 years old (mean 44 years old). They all had $10 / 10$ or corrected to $10 / 10$ vision, held a valid driving license for almost 2 years (mean 25 years) and drove $26000 \mathrm{~km} /$ year on average. 


\section{Driving simulator}

This experiment was carried out using the SAAM dynamic driving simulator (referred to previously as SAM (Colombet et al, 2009), see Figure 3). It is composed of a cockpit based on a Renault Twingo II standard car which has been lightened and instrumented. The inside of the cockpit is unchanged so it is visually identical to the initial car.

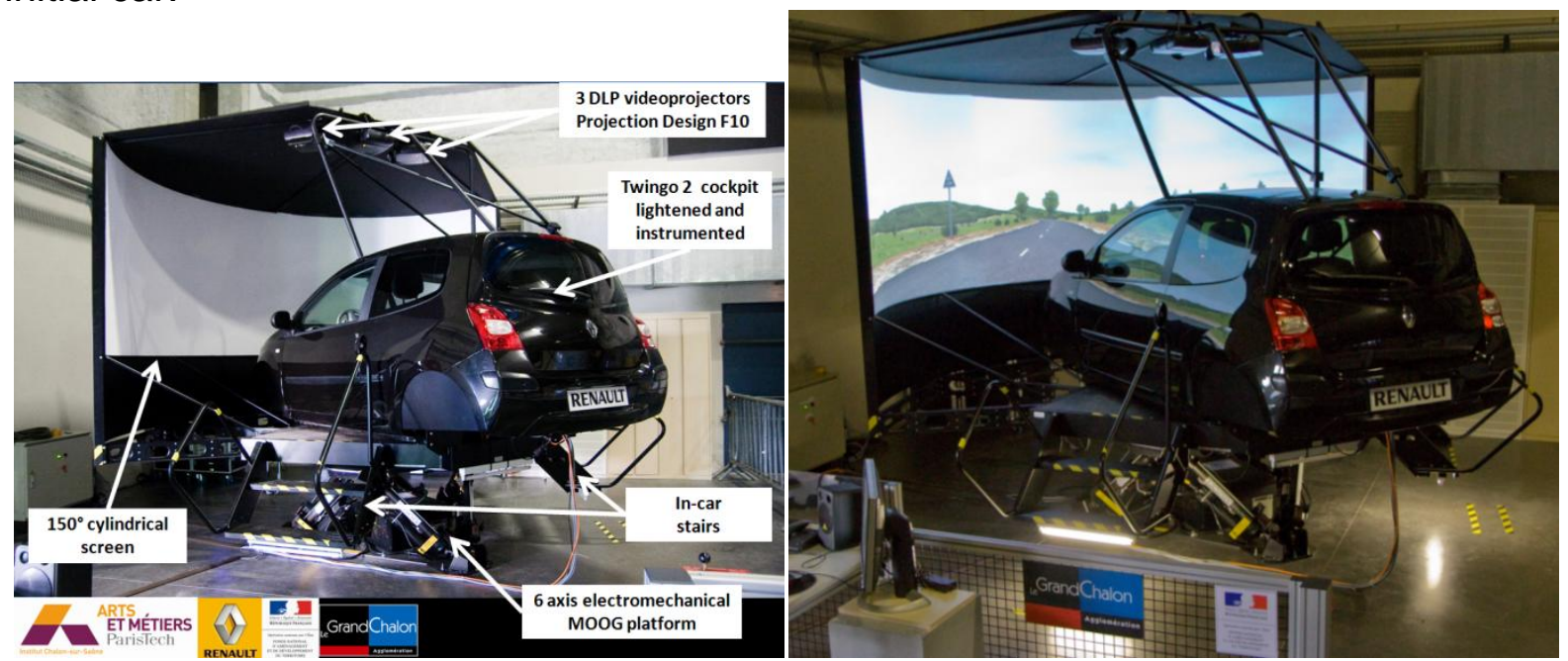

Figure 3 - SAAM dynamic driving simulator (Arts et Métiers ParisTech / Renault)

Visual environment is projected thanks to 3 DLP projectors at a $1280 \times 1024$ resolution per channel on a $150^{\circ}$ cylindrical screen. Accelerations are rendered thanks to a Gough-Stewart electromechanical platform (MOOG $2000 \mathrm{E}$ ) which allows 6 degrees of freedom ( $\left.\pm 20 \mathrm{deg}, \pm 0.25 \mathrm{~m}, \pm 5 \mathrm{~m} / \mathrm{s}^{2}\right)$. A classical motion cueing algorithm with anti-backlash filters (Reymond \& Kemeny, 2000) is used to compute the simulator displacements. However simulated vehicle vibrations are not rendered.

Haptic rendering is done on the steering wheel (active electromechanical system) and on the pedals (passive mechanical system). The gearbox is the original five speed automatic gearbox of the car. Sound of the engine, the road and the traffic is rendered through the cockpit speakers located in the doors. Surrounding parasite sounds (such as actuators noise) are cut off thanks to the cockpit which is completely closed. An intercom facility yet allows for communication between the cockpit and the control room. The whole driving simulation is generated by the SCANeR@ II software (Oktal, Renault).

\section{Experimental conditions}

Five visual scale factors were compared: $0.70,0.85,1.00,1.15$ and 1.30 . Once again in every case the computed image is displayed on the whole screen. In this way the driver's field of view covered by the virtual scene remains identical during all the experiment. Figure 4 presents screenshots from central display with different visual scale factors, showing the effect on the computed image. The car is always at the same position for all these screenshots. 


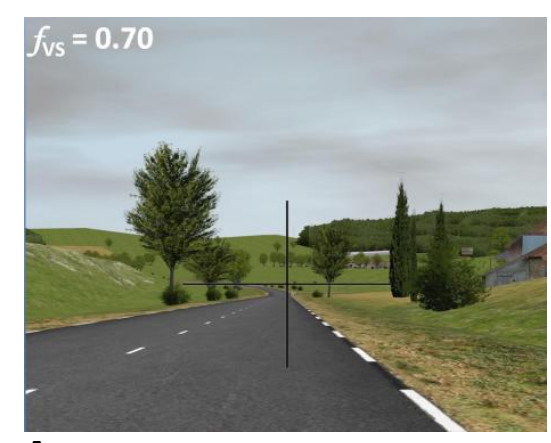

A

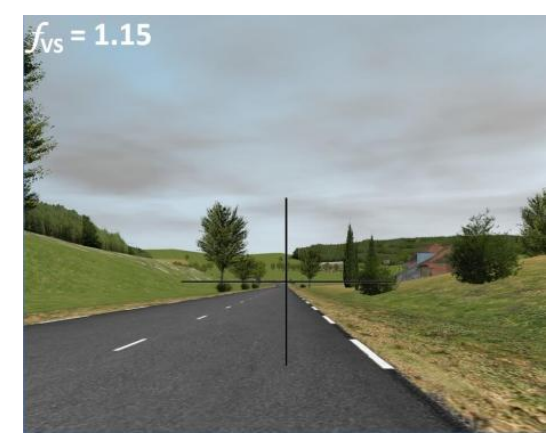

\section{D}

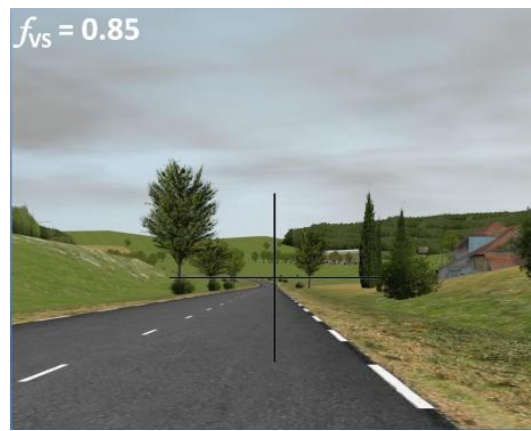

B
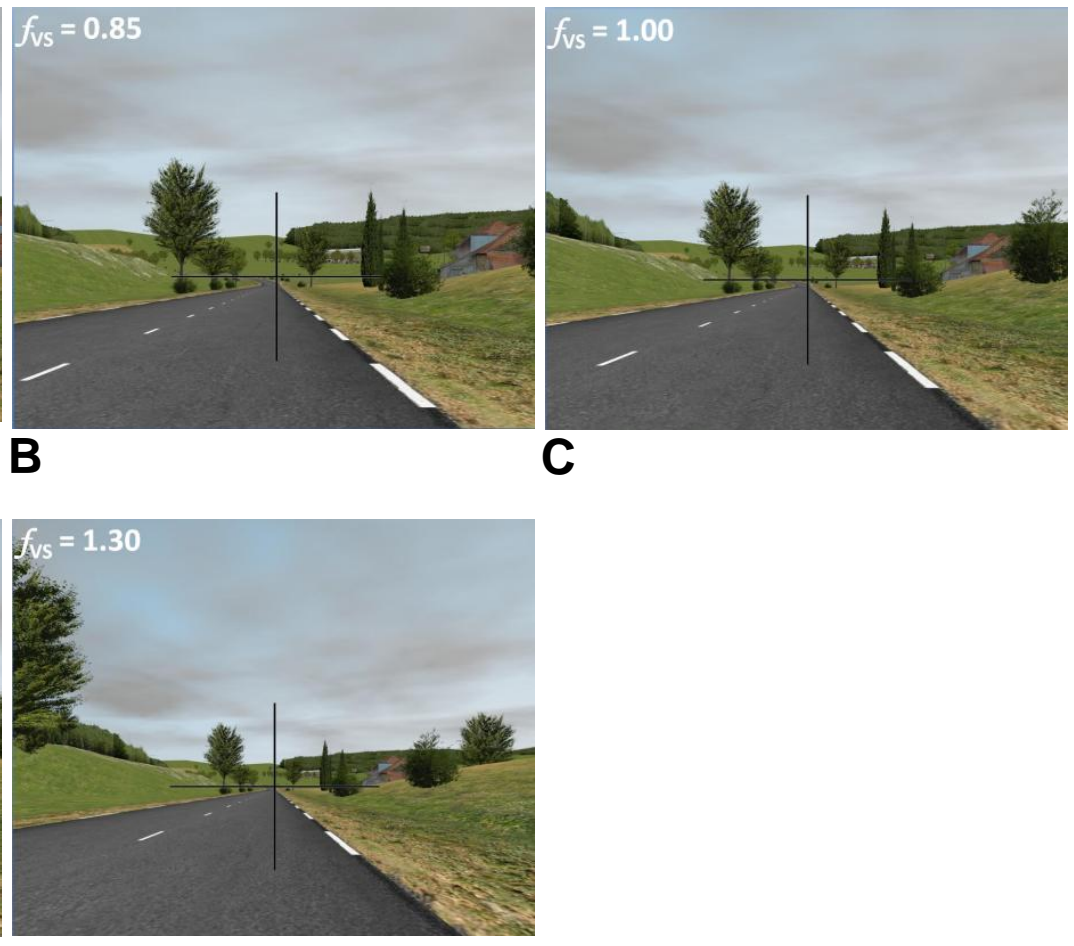

E

Figure 4 - Screenshots taken with visual scale factors of $0.70,0.85,1.00,1.15$ and 1.30 in respectively $A, B, C, D$ and $E$. All these screenshots correspond to only the center image displayed in the simulator (corresponding to $52^{\circ}$ of driver's horizontal field of view).

\section{Experimental protocol}

After a free practice drive to familiarize with the driving simulator, subjects were asked to reproduce two speeds $(50 \mathrm{~km} / \mathrm{h}$ and $90 \mathrm{~km} / \mathrm{h})$ in the 5 different visual conditions. The experiment took place on a straight country road (see screenshots Figure 4).

For each speed and for each visual condition, subjects drove two times. First they drove with a speed regulator (cruise control) at the consign speed. Speedometer was hidden so they didn't know the numerical value of this speed and thus no bias was introduced. The visual scale factor was then of 1.00. This first driving session lasted about $1 \mathrm{~min}$.

For the second driving session, the speed regulator was disabled and the visual scale factor was randomly changed. Subjects were asked to reach the speed at which they were the first time and then to act the turn signal. The reached speed at which they felt like at consign speed was measured as soon as the turn signal was activated. Besides, as sound plays also an important role in speed perception (Kemeny \& Panerai, 2003), it was disabled. Speed perception is analyzed through the speed reached by the subjects. Actually the more the speed perception grows the more the speed reached will decrease for the same perceived speed.

Each participant tested every visual scale factor with every consign speed. The order of treatment of these 10 conditions was random. Table 1 summarizes the simulator configurations for the 2 driving sessions repeated by the subject 10 times (one for each configuration in random order). 


\begin{tabular}{|l|l|l|}
\hline & $\mathbf{1}^{\text {st }}$ driving session & $\mathbf{2}^{\text {nd }}$ driving session \\
\hline Speed consign & $50 \mathrm{~km} / \mathrm{h}$ or $90 \mathrm{~km} / \mathrm{h}$ & $\begin{array}{l}\text { Same as in first driving } \\
\text { session }\end{array}$ \\
\hline Visual scale factor $f_{\text {sv }}$ & 1.0 & $\begin{array}{l}0.70,0.85,1.00,1.15 \text { or } 1.30 \\
\text { randomly }\end{array}$ \\
\hline Speed regulator & $\begin{array}{l}\text { Enabled: forced to speed } \\
\text { consign (not piloted by driver) }\end{array}$ & Disabled \\
\hline Sound & Disabled & Disabled \\
\hline Speedometer & Hidden & Hidden \\
\hline $\begin{array}{l}\text { Motion rendering / haptic } \\
\text { rendering }\end{array}$ & Enabled & Enabled \\
\hline
\end{tabular}

Table 1 - For each of the 10 conditions ( 2 different consigns of speed and 5 different visual scale factors), subject had to drove 2 times. This table summarizes the simulator configurations for these two driving sessions.

\section{Results}

The Figure 5 presents the speeds reached by the participants as a function of the visual scale factor. Values are sorted by corresponding speed consign $(50 \mathrm{~km} / \mathrm{h}$ in blue circles and $90 \mathrm{~km} / \mathrm{h}$ in red triangles). Left graph presents all the values and right graph presents the means and the error bars representing $95 \%$ confidence level.
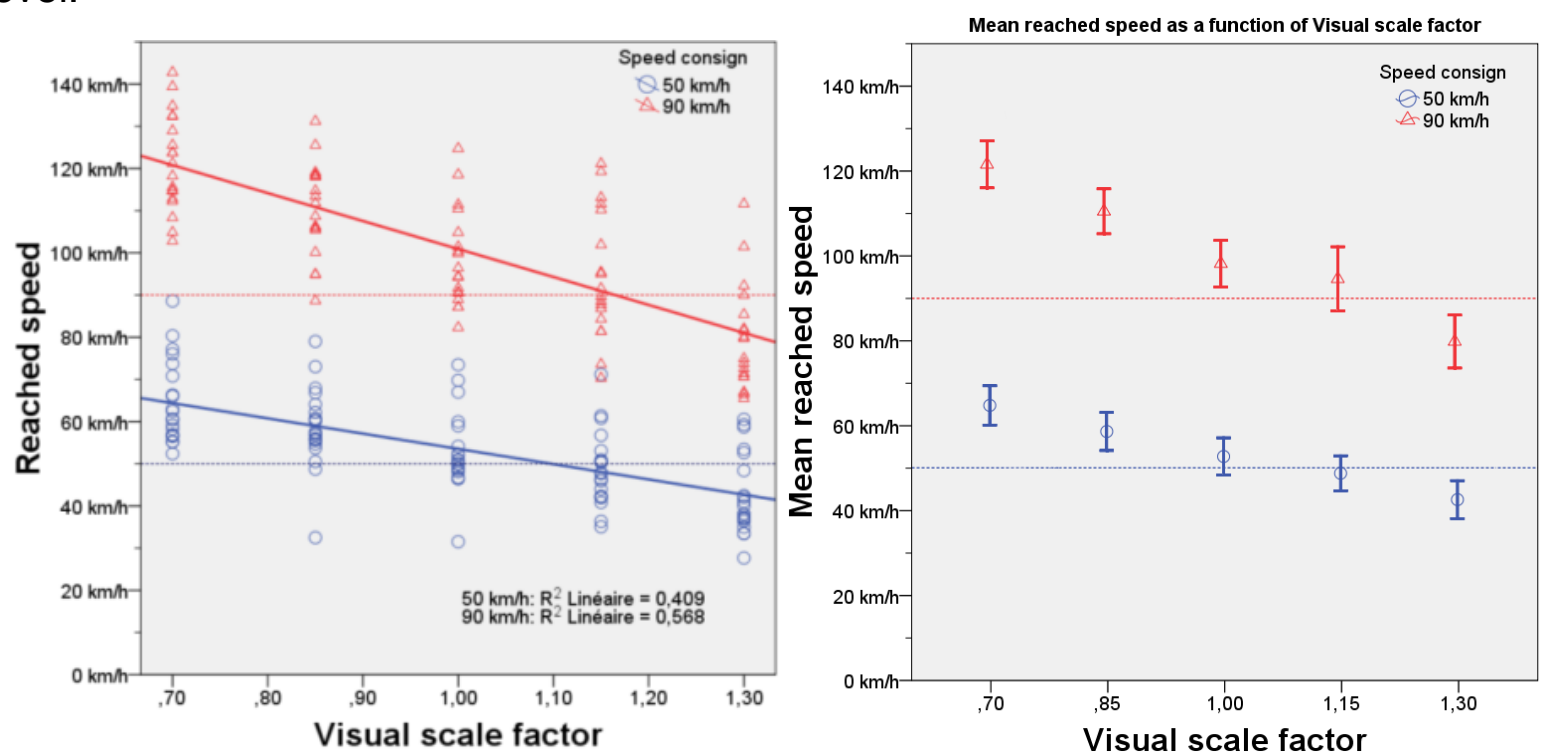

Figure 5 - Reached speed (on the left) and mean reached speed (on the right) as functions of visual scale factor for both speed consigns $(50 \mathrm{~km} / \mathrm{h}$ in blue circles and $90 \mathrm{~km} / \mathrm{h}$ in red triangles). Vertical error bars represent $95 \%$ confidence level.

We can see on these graphs that as expected, speed reached by the participants is decreasing while the visual scale factor is increasing. It actually means that perceived speed is increasing with the visual scale factor. This decrease is very linear. Regressions have been done and the $\mathrm{R}^{2}$ coefficients corresponding to the 50 $\mathrm{km} / \mathrm{h}$ and the $90 \mathrm{~km} / \mathrm{h}$ consign are respectively 0.409 and 0.568 . Furthermore, the slope of the linear regression seems to be proportional to the consign speed. In the Figure 6 we draw this slope as a function of the consign speed and we also assume that for a $0 \mathrm{~km} / \mathrm{h}$ consign, subjects would always reproduce a null speed, i.e. the slope is null. We thus obtain a very good linear regression with $R^{2}=0.999$. We can 
then conclude that the variation of perceived speed generated by the visual scale factor is proportional to the actual speed of the virtual vehicle.

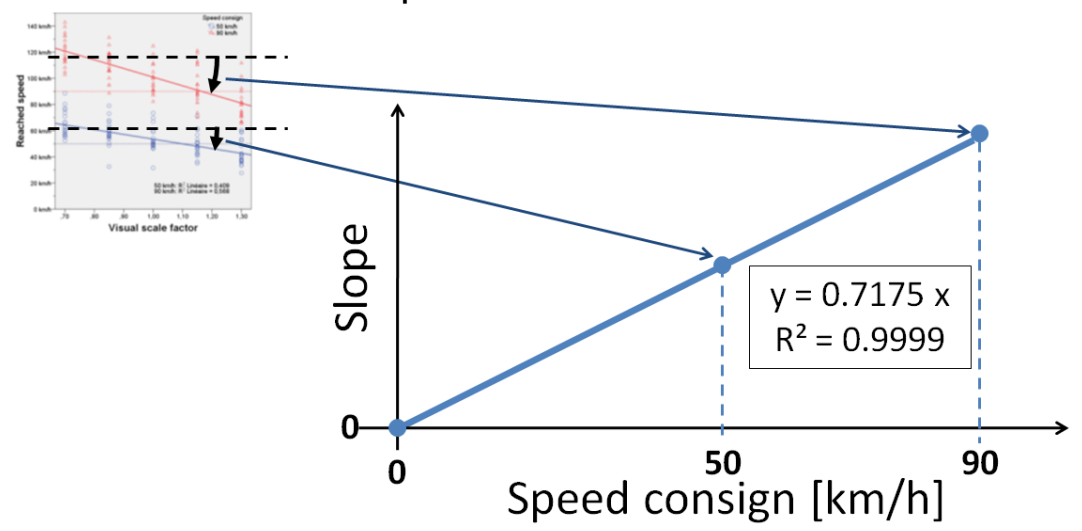

Figure 6 - The variation of produced speed (and of perceived speed) generated by the visual scale factor is proportional to the speed consign.

This finding allows us to study our data all together relatively to speed consign. The error of produced speed is shown in Figure 7 with the obtained values (on the left) and the corresponding mean errors (on the right) drawn as functions of the visual scale factor. We can see that error is also decreasing along a linear function $\left(\mathrm{R}^{2}=\right.$ $0.469)$ while the visual scale factor is increasing.
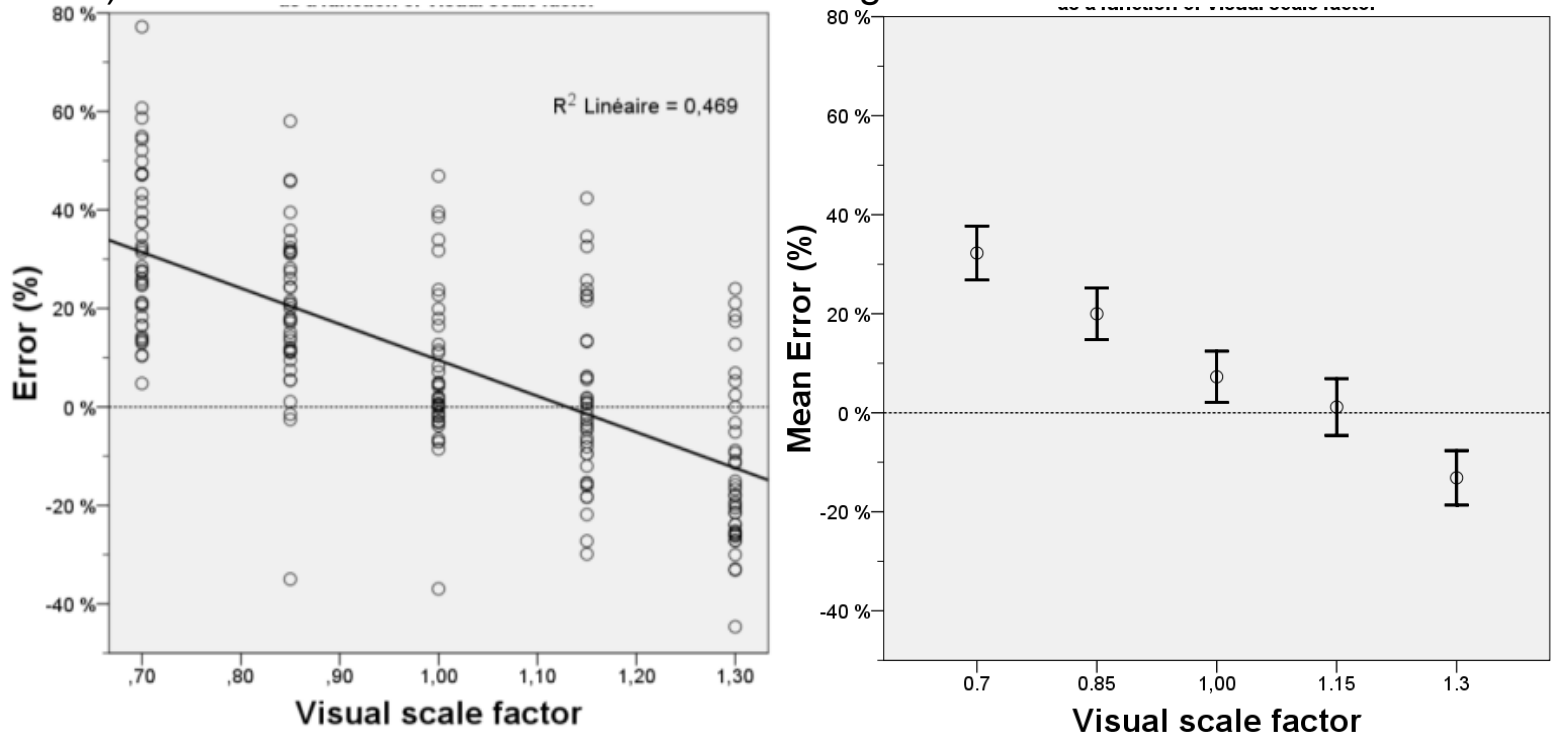

Figure 7 - Error (on the left) and mean error (on the right) relative to speed consign displayed as functions of visual scale factor. Vertical error bars represent $95 \%$ confidence level.

In order to study individual results, we also computed for each data the error relative to the speed reached by the same subject for the same speed consign with the visual scale factor of 1.0. We obtained the results presented in Figure 8 where we can see that this error is decreasing along the linear function $y=-68.4 *(x-1)$ with $\mathrm{R}^{2}=0.54$. We can also see that there is almost no negative value for 0.70 and 0.85 visual scale factors (respectively $5 \%$ and $3 \%$ ) whereas there are some positive values for 1.15 and 1.30 visual scale factors (respectively $33 \%$ and $10 \%$ ). That means that relatively to the speed that subjects reached with visual scale factor of 1.0 , they drove faster in $96 \%$ of the case with 0.70 and 0.85 factors. But with 1.15 and 1.30 factors, subjects drive more slowly in only $79 \%$ of the case. 

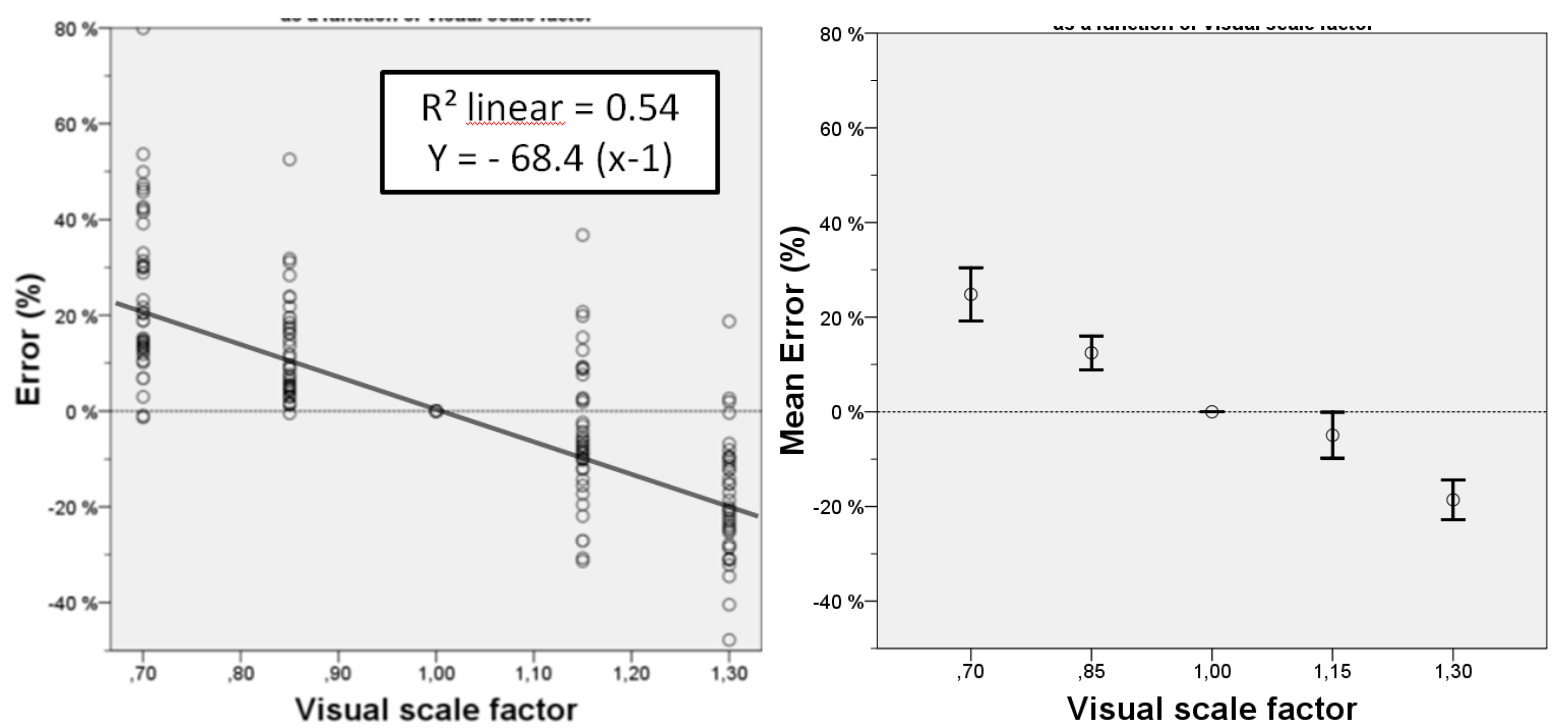

Figure 8 - Error (on the left) and mean error (on the right) relative to speed reached by the same subject with the same speed consign with a visual scale factor of 1.0, as functions of visual scale factor. Vertical error bars represent $95 \%$ confidence level.

A one-factor ANOVA with Tukey post hoc tests was used to analyze these error data. It showed that visual scale factor is highly significant with $p<0.001$. Post hoc results show significant differences between each visual scale factor $(p<0.001)$ except between 1.00 and 1.15 for which we obtain $p=0.431$.

To the question: "Did you notice any variation in the simulator settings between the different driving sessions?", all the subjects answered they did not.

\section{Conclusion and perspectives}

We first showed how the modification of the geometric field of view alters the optic flow. It explains why the visual scale factor, which corresponds to the ratio between the GFOV and the FOV, affects the perception of speed. We showed that for a speed reproduction task, visual scale factor modifications significantly impacted the speed reached by the subjects, which means that speed perception increases with the visual scale factor. We also showed that a modification of 0.15 of the visual scale factor was enough to obtain a highly significant impact $(p<0.001)$ on speed perception. Furthermore, these visual modifications seem to be subtle enough to remain unnoticed by drivers. So this technique can easily be employed to have drivers reduce or increase their speed in driving simulation conditions. These first conclusions are consistent with those obtained by (Mourant et al, 2007) and (Diels \& Parkes, 2009) though the task (speed reproduction instead of speed production) and the simulation conditions (dynamic with 6 DOF instead of respectively no motion and only vibrations) were different.

We also showed that the impact of the visual scale factor was proportional to the speed of the virtual vehicle. According to Figure 8 , we can quantify the variation of perceived speed generated by the visual scale factor with the empirical law:

$$
\Delta_{\text {perceived speed }}(\%) \approx 68 *\left(f_{V S}-1\right)
$$

For example the perceived speed with a visual scale factor of 1.15 will be $68 *(1.15-1) \approx 10 \%$ higher than perceived speed with a visual scale factor of 1.00 . Conversely perceived speed will be $10 \%$ smaller with a visual scale factor of 0.85 . 


\section{Perspectives}

We have seen that GFOV modification has an impact on speed perception and seems to remain unnoticed by drivers. And as speed perception is often underestimated in virtual reality applications (Banton et al, 2005), using the visual scale factor could be used for dedicated driving simulators, especially for low cost driving simulators vs. full scale driving simulators. With the above-presented law, one can easily find which visual scale factor to use in order to improve the speed perception rendering of his/her simulator.

However, the difference between the actual speed and the perceived speed seems also to depend on the speed according to (Mourant et al, 2007). So the visual scale factor should be dynamically changed as a function of the vehicle speed. Yet effects of dynamic variations of the visual scale factor have not been investigated and knowing the necessary conditions to keep these variations unnoticed by the drivers seems necessary. Furthermore, as acceleration is mathematically the speed derivative, dynamic variations of the GFOV may also have an effect on acceleration perception.

We have also seen that GFOV modification seems to remain unnoticed by drivers. However, visual scale factor may also affect perception of distances. For example in Figure 4, the same tree on the left of the road seems to be nearer with a 0.70 visual scale factor than with a 1.30 factor. Comparing driver's behavior in tasks like following a vehicle or emergency braking while using different visual scale factors could allow understanding better the real impact of this technique. A presence questionnaire could also be used in this experiment in order to verify that the driver's immersion and presence are not affected by the visual scale factor.

\section{Acknowledgements}

This work has been done thanks to the support of Le Grand Chalon.

\section{References}

Banton, T., Stefanucci, J., Durgin, F., Fass, A., \& Proffitt, D. (2005). The perception of walking speed in a virtual environment. Presence: Teleoperators and Virtual Environments , 14 (4), 394-406.

Berthoz, A., Pavard, B., \& Young, L. R. (1975). Perception of linear horizontal self-motion induced by peripheral vision (linearvection) basic characteristics and visual-vestibular interactions. Experimental Brain Research , 23, 471-489.

Berthoz, A. (1995). Le sens du mouvement. Ed. Odile Jacob

Colombet, F., Kemeny, A., Mérienne, F., \& Père, C. (2009). Motion cueing strategies for driving simulators. Proceedings of the WINVR 09 Conference. Chalonsur-Saône, France.

Diels, C., \& Parkes, A. M. (2009). Geometric Field Of View Manipulations Affect Perceived Speed in Driving Simulators. Proceedings of the Road Safety and Simulation Conference. Paris, France.

Jamson, A. H. (2000). Driving Simulator Validity: Issues of Field of View and Resolution. Proceedings of the Driving Simulation Conference, 57-64. Paris, France.

Kemeny, A., \& Panerai, F. (2003). Evaluating perception in driving simulation experiments. TRENDS in Cognitive Science , 7 (1), 31-37. 
Kennedy, R. S., Lane, N. E., Berbaum, K. S., \& Lilienthal, M. G. (1993). Simulator Sickness Questionnaire: An Enhanced Method for Quantifying Simulator Sickness. The International Journal of Aviation Psychology , 3, 203-220.

Lappe, M., Bremmer, F., \& van den Berg, A. V. (1999). Perception of selfmotion from visual flow. Trends in Cognitive Sciences , 3 (9), 329-336.

Lee, David N. (1976) A theory of visual control of braking based on information about time-to-collision. Perception, 5: 437-459.

Mourant, R. R., Ahmad, N., Jaeger, B. K., \& Lin, Y. (2007). Optic flow and geometric field of view in a driving simulator display. Displays , 28, 145-149.

Panerai, F., Droulez, J., Kelada, J. M., Kemeny, A., Balligand, E., \& Favre, B. (2001). Speed and safety distance control in truck driving: comparison of simulation and real-world environment. Proceedings of the Driving Simulation Conference, 91108. Sophia Antipolis (Nice), France.

Reymond, G., \& Kemeny, A. (2000). Motion cueing in the Renault driving simulator. Vehicle System Dynamics, 34, 249-259. 